\title{
Uso de nuevas tecnologías en el proceso electoral hondureño: voto electrónico, análisis de percepción en la UNAH-VS, febrero-mayo de 2013
}

Larissa Josselin Murcia Rápalo, Jack Antonio Avilez Alvarado, Noé Jesús Guerra, Daniel Irías Licona, Adonaldo Alvarado Henrríquez ${ }^{1}$

Mirta Maribel Macías Ruano²

\section{RESUMEN}

La sociedad del conocimiento caracterizada por avances tecnológicos progresa de manera acelerada y abarca los distintos ámbitos de nuestra vida. En tal escenario, el ámbito político también se ve inmerso en este proceso, de manera que el uso de nuevas tecnologías en los procesos electorales es perfila para el futuro en muchos países, pero es el presente en otros que ya han aplicado dicha opción en su proceso electoral.

La presente investigación titulada: Uso de nuevas tecnologías en proceso electoral hondureño: voto electrónico, análisis de percepción en la UNAH-VS, febrero-junio de 2013, nace de la efervescencia política que se vive en Honduras debido, entre otras razones, al debate sobre la implementación del voto electrónico en el proceso electoral 2013 y es el resultado del trabajo investigativo realizado por estudiantes de la asignatura de Estadística Económica l.

El objetivo de la investigación es identificar el nivel de aceptación del uso de nuevas tecnologías (voto electrónico) en el proceso electoral 2013, entre los estudiantes de la UNAH-VS. La misma es de carácter descriptivo a partir de los resultados obtenidos. Los resultados obtenidos, entre otros, indican que el $90 \%$ de la población estudiantil ha escuchado hablar del voto electrónico y sabe en qué consiste esta opción de votación. Los entrevistados en un $68 \%$ dan una valoración de confianza al Tribunal Supremo Electoral, lo cual está en el rango de entre 0-50\%; de igual manera, un $92 \%$ considera que debe modernizarse el actual sistema de votación.

Palabras clave: democracia, política, elecciones, nuevas tecnologías y voto electrónico.

\footnotetext{
${ }^{1}$ Estudiantes de Economía de la UNAH-VS.

${ }^{2}$ Asesora, Coordinación Regional de Investigación UNAH-VS. Dirección electrónica: mirtamaru@hotmail.com
} 


\section{ABSTRACT}

The knowledge society characterized by technological advances progresses rapidly, and covers various areas of our life. In such a scenario, the political area is also immersed in this process, so that the use of new technologies in the electoral process is available for the future in many countries, but is present in others who have already implemented this option in their electoral process. The present study titled : Using new technologies in Honduran elections : electronic voting , discussion of its perception in UNAH -VS , February- June 2013, was born from the political turmoil that exists in Honduras because, among other reasons, the debate on the implementation of electronic voting in the 2013 election. The objective of the research is to identify the level of acceptance of the use of new technologies (e-voting) in the 2013 electoral process, taking in consideration the opinion of UNAH -VS students' .The results indicate that $90 \%$ of the student population has heard of electronic voting and know about this voting option. $68 \%$ of the interviewed people give a $68 \%$ confidence rating to the Supreme Electoral Tribunal, and $92 \%$ think that the current voting system must be modernized.

Key Words: Democracy, politics, elections, new technologies and e-voting

\section{INTRODUCCIÓN}

En el presente trabajo expone de manera resumida los resultados obtenidos en la investigación realizada en el marco de la asignatura de Estadística Económica I, referente al tema: Uso de nuevas tecnologías en proceso electoral hondureño: voto electrónico, análisis de percepción en la UNAH-VS, febrero-mayo de 2013.

Una de las motivaciones para realizar dicha investigación, entre otras razones, radica en el hecho de que era un tema de debate actual, por estar en un año electoral, la cual se considera como un punto de partida para futuras investigaciones sobre la temática, porque sigue teniendo vigencia actualmente. Asimismo, hay que destacar que se aporta información primaria que se obtuvo a partir de las encuestas aplicadas a los estudiantes.

El artículo se ha estructurado de la siguiente manera: un primer apartado dedicado a la introducción, en el segundo se aborda el método utilizado para realizar la investigación, un tercer segmento se dedica a los resultados, en el cuarto se expone la discusión y finalmente se proporcionan las conclusiones. 


\section{METODOLOGÍA}

Antes de la descripción metodológica, se precisa plantear el problema. En tal sentido, el uso de nuevas tecnología en el proceso electoral presenta una serie de interrogantes debido a los desafíos que plantea, sin embargo, por ser un tema reciente en Honduras, nos interesa conocer lo siguiente: ¿Cuál es el conocimiento que tiene el estudiante sobre el voto electrónico? ¿Cuál es el nivel de aceptación del uso del voto electrónico en el proceso electoral?

A partir de las preguntas/problemas, se plantearon las hipótesis siguientes:

1. Existe un alto nivel de conocimiento del voto electrónico entre los estudiantes.

2. Hay un alto nivel de aceptación en el uso de nuevas tecnologías en el proceso electoral.

La metodología utilizada en la realización de la investigación es de tipo cuantitativa, con carácter descriptivo, debido a que se analizó cómo es y cómo se manifiesta la percepción que los estudiantes de UNAH-VS tienen sobre la implementación de las nuevas tecnologías en el proceso electoral hondureño.

Con la finalidad de recolectar información sobre la percepción que tiene el estudiantado de la UNAH-VS sobre el voto electrónico, se aplicó una encuesta en el área económica-administrativa aproximadamente a 200 estudiantes. Los criterios de inclusión para la muestra fueron los siguientes:

1. Estar cursando actualmente una asignatura.

2. Asistir regularmente a la clase, se solicitó colaboración de los docentes al respecto.

3. Ser mayor de edad.

4. Para evitar duplicidad al ser seleccionado, se les pidió el número de cuenta y si era necesario se excluía de la base de datos.

Los criterios de exclusión fueron los siguientes: ser menor de edad, no estar matriculado y, en caso de estar matriculado, no asistir regularmente (listado de docentes). 
El instrumento aplicado, o sea la encuesta, tenía por objetivo recolectar información referente a aspectos tales como el conocimiento del voto electrónico, principales ventajas del voto electrónico, confianza de las instituciones relacionadas con el proceso electoral. Una vez elaborado el instrumento, se procedió a validar el mismo mediante una prueba piloto, con la finalidad de identificar sus inconsistencias y para cronometrar el tiempo de ejecución para hacer una relación de la productividad por encuestador y el costo de cada encuesta.

Finalmente, obtenida la información, se procedió a la validación en la base de datos en campo, la cual se realizó a través de la verificación de los filtros y pases, por tanto, el bloque de respuestas debe estar en blanco. En la base, el proceso incluyó la consistencia de la base de datos a través de los principales medios estadísticos usados, los valores $Z$ dispersos, valores missing, etc.

\section{RESULTADOS}

En términos generales, las valoraciones que se deducen indican que aproximadamente un $90 \%$ de la población entrevista ha escuchado hablar del voto electrónico y que un $88 \%$ de los entrevistados considera oportuno modernizar el sistema electoral del país, en tanto que el $56 \%$ señala que en el país, ninguna área está preparada para la implementación del sistema de voto electrónico; por el contrario, un $26 \%$ considera que económicamente sí existen tales condiciones (ver cuadro 1 y gráfico 1).

Cuadro 1. ¿Ha escuchado hablar del voto electrónico?

\begin{tabular}{|l|c|}
\hline Sí & $90 \%$ \\
\hline No & $10 \%$ \\
\hline Total & $100 \%$ \\
\hline
\end{tabular}


Gráfico 1. Considera que el país está más preparado para la implementación del voto electrónico

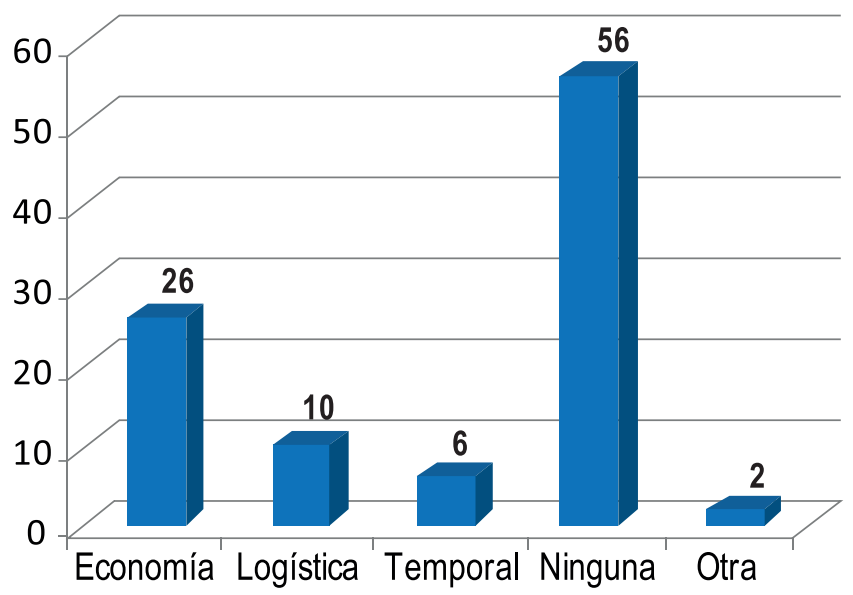

Estos porcentajes reflejan importante información debido a que la encuesta se desarrolló en un grupo estudiantil del área económica-administrativa, derivada tal percepción del grado de conocimiento político, social y económico del país que se desarrolla al interior de esta Universidad.

Según la información recabada (ver gráficos 2 y 3 ), los principales resultados que se obtienen con la implementación de nuevas tecnologías son reducción de costos (24 $\%$ ), resultados en tiempo real (22\%) y transparencia (22\%).

Grafico 2. Porcentaje de confiabilidad que le asigna al actual sistema de votación

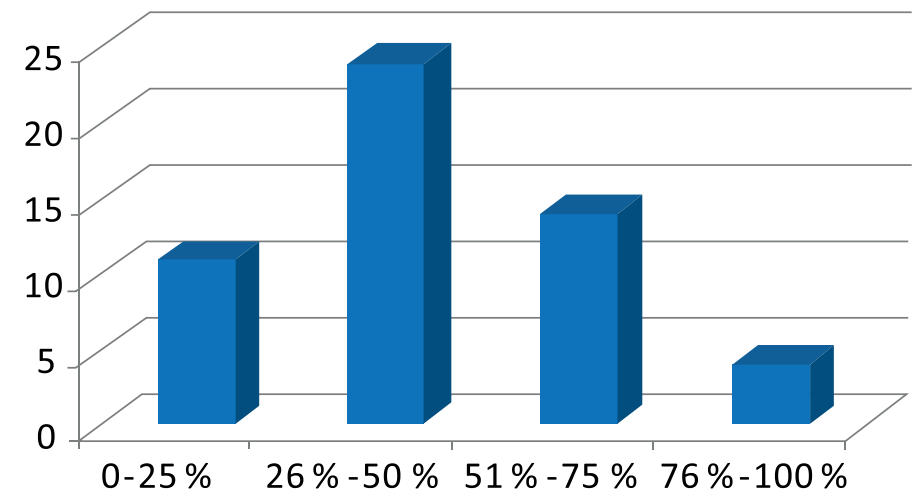

Fuente: Elaboración propia con base a encuestas VE 2013. 
Gráfico 3. Cuáles pueden ser los mayores beneficios del voto electrónico

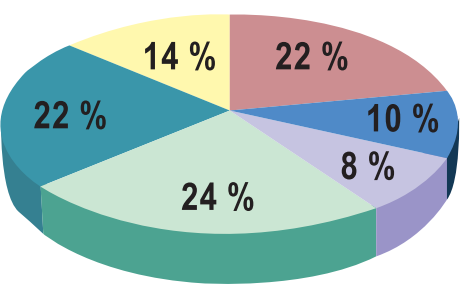

Resultados en tiempo real/ mayor transparencia

- Resultados en tiempo real/ reducción de costos Resultados en tiempo real/ modernización del sistema de votación

- Mayor transparencial reducción de costos

- Mayor transparencia/ modernización del sistema de votación

- Reducción de costos/modernización del sistema de votación

Fuente: Elaboración propia con base a encuestas VE 2013.

Con relación a lo anterior, los entrevistados consideran que con la implementación de nuevas tecnologías en las elecciones se reducirían los conflictos al momento de la presentación de los resultados (46\%). Asimismo, el 78 \% respondió que es oportuno que se le consulte e informe al pueblo sobre la implementación de nuevas tecnologías en el proceso electoral (ver gráficos 4 y 5 ).

Gráfico 4. ¿Cree usted que la implementación del voto electrónico reducirá los conflictos al momento de presentar los resultados?

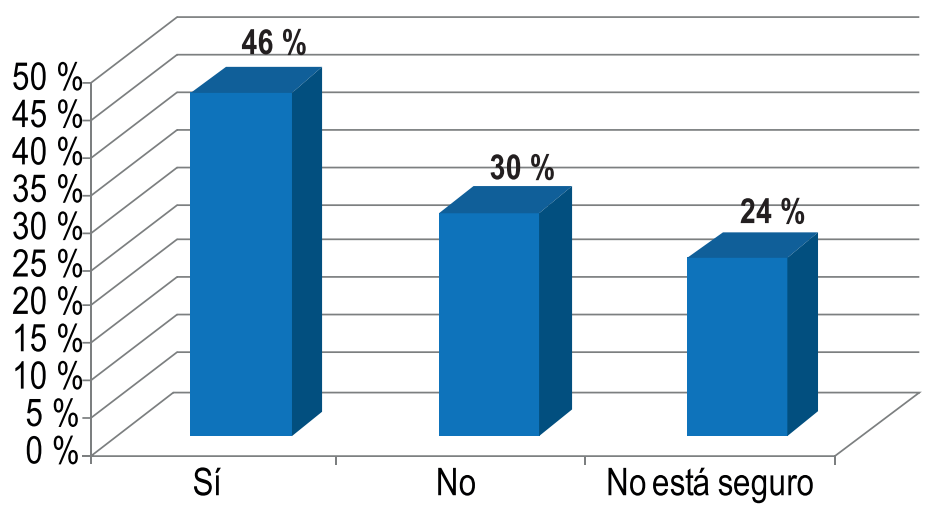

Fuente: Elaboración propia con base a encuestas VE 2013. 
Gráfico 5. Considera oportuno que se consulte al pueblo hondureño sobre la implementación de el voto electrónico



Fuente: Elaboración propia con base a encuestas VE 2013.

\section{CONCLUSIONES}

Partiendo de los resultados obtenidos, se considera oportuno usar nuevas tecnologías en el proceso electoral. De igual manera, se identificó que los estudiantes de la UNAH-VS están muy familiarizados con el concepto de nuevas tecnologías, concretamente con el voto electrónico, de esta forma se comprueban las hipótesis planteadas.

No obstante, por ser un proceso reciente y debido a que el tema sale a debate en este año político, se considera que es prematura la implementación del voto electrónico, porque no hay la suficiente preparación institucional ni del recurso humano. $Y$, en caso de implementarse, los principales beneficios serán la transparencia y los resultados en tiempo real.

Cabe mencionar que con la presente investigación se aporta información importante sobre el uso del voto electrónico, por lo que se puede considerar a esta información como un punto de partida para investigaciones sobre la temática, por la relevancia social que ha adquirido el tema en una época de elecciones en Honduras.

\section{AGRADECIMIENTO}

Agradecemos a la docente Mirta Macías, de la clase de Estadística Económica I, por su colaboración brindada en el transcurso de la investigación; igualmente a todas las personas que de alguna manera contribuyeron a la realización de esta tarea. 


\section{BIBLIOGRAFÍA}

Organización Mundial de la Salud. (Julio-diciembre de 2012). Democracia, elecciones y nuevas tecnologías. El voto electrónico. Revista Mexicana de Análisis Político yAdministración Pública, (1), 2, pp. 9-21.

Pineda, B.E; Alvarado, E. Ly Canales, H.F. Metodología de la investigación.

Prince, A; Jolias, J; Lacabanne, F. et al. (2012). Voto electrónico en Argentina. Anales del SIES, pp. 73-85.

Tula, Ml; Pegora y Zulcovsky, M. (2005). Voto electrónico: entre votos y máquinas. Las nuevas tecnologías en los procesos electorales. Buenos Aires: Ariel. 